Artigo

\title{
Análise de Tendências em Dados de Precipitação e Temperatura no Brasil
}

\author{
Marcos Figueiredo Salviano ${ }^{1}$, Juliano Daniel Groppo ${ }^{2}$, Giampaolo Queiroz Pellegrino ${ }^{3}$ \\ ${ }^{1}$ Companhia de Pesquisa de Recursos Minerais, São Paulo, SP, Brasil. \\ ${ }^{2}$ Escola de Engenharia de Piracicaba, Piracicaba, SP, Brasil. \\ ${ }^{3}$ Embrapa, Campinas, SP, Brasil.
}

Recebido: 17/5/2014 - Aceito 5/5/2015

\begin{abstract}
Resumo
A concentração de gases de efeito estufa (GEE) na atmosfera tem aumentado acentuadamente desde a revolução industrial, o que levou a intensificação do efeito estufa e consequentemente vem causando o aquecimento global. A análise espacial de tendências permite observar as mudanças no comportamento e determinar em quais regiões uma determinada variável vem sofrendo mudanças ao longo do tempo. Diante disso, o objetivo do presente trabalho foi analisar as tendências temporais da precipitação e da temperatura média no Brasil, utilizando o método Contextual Mann-Kendall (CMK), utilizando dados espacialmente distribuídos elaborados pelo Climatic Research Unit (CRU), entre os anos 1961 e 2011. A umidade relativa e a evapotranspiração foram analisadas no intuito de auxiliar na interpretação dos resultados da precipitação e temperatura. Os resultados mostraram tendências não significativas em mais de $70 \%$ do território brasileiro em todos os meses na precipitação, porém a temperatura média apresentou tendência positiva significativa em grande parte do Brasil ao longo de todo ano. Em geral, a evapotranspiração apresentou um comportamento diretamente proporcional à temperatura, enquanto que a umidade relativa apresentou comportamento inversamente proporcional. A continuidade dessas tendências poderá resultar em impactos na agricultura e no ciclo hidrológico, e consequentemente para a fauna e flora e para a população.
\end{abstract}

Palavras chave: análise de tendências, Mann-Kendall, variáveis meteorológicas, SIG.

\section{Trends Analysis of Precipitation and Temperature Data in Brazil}

\begin{abstract}
The concentration of greenhouse gases (GHGs) in the atmosphere has increased dramatically since the industrial revolution, which led to intensification of the greenhouse effect and thus global warming. The spatial trend analysis allows us to observe changes in behavior and determine which region a given variable has suffered significant changes over time. Therefore, the aim of this study was to analyze trends in time series to rainfall and mean temperature in Brazil using the Contextual Mann-Kendall method (CMK), using data produced by spatially distributed Climatic Research Unit (CRU), between 1961 and 2011. The relative humidity and potential evapotranspiration were used to help understand the results. The results showed no significant trends in over $70 \%$ of the Brazilian territory in all months in the precipitation, but the mean temperature showed a significant positive trend in much of Brazil throughout the year. In general, evapotranspiration presented a behavior directly proportional to the temperature, while the relative humidity had an inversely proportional behavior. The continuation of these trends could result in impacts on population, agriculture, water cycle, fauna and flora.
\end{abstract}

Keywords: trend analysis, Mann-Kendall, meteorological variables, GIS.

\section{Introdução}

A concentração de gases de efeito estufa (GEE) na atmosfera tem aumentado acentuadamente desde a revolução industrial, o que levou a intensificação do efeito estufa e consequentemente vem causando o aquecimento global
(IPCC, 2007). Os impactos associados às mudanças climáticas serão sentidos em todo o mundo e, provavelmente, terão implicações profundas para a população humana (Nobre et al., 2011).

Os cenários de mudanças climáticas apontam para um aumento acima de $2{ }^{\circ} \mathrm{C}$ na temperatura média global

Autor de correspondência: Marcos Figueiredo Salviano, marcosfs123@gmail.com. 
(IPCC, 2007). Esse aumento acarretará vários impactos nos ecossistemas e no ciclo hidrológico, com alteração nos padrões de chuva e temperatura, além da intensificação de eventos climáticos extremos. Podendo causar impactos em diversos segmentos socioeconômicos do Brasil, como a agricultura e pecuária (Pinto et al., 2001), geração de energia (de Lucena et al., 2010), abastecimento de água e a ocorrência de desastres naturais (Marengo, 2010), impactos significantes na fauna e flora (PBMC, 2013).

Além da mudança do clima, outros fatores contribuem para o aumento da vulnerabilidade hídrica brasileira, entre eles: a pressão demográfica, o crescimento urbano desordenado, a pobreza e a migração rural, e o baixo investimento em infraestrutura e serviços.

A análise espacial de tendências permite observar as mudanças no comportamento e determinar em quais regiões uma determinada variável vem sofrendo mudanças significativas ao longo do tempo. Mudanças que podem estar relacionadas a diversos fatores, de origem antrópica como desmatamentos e urbanização desenfreada (Serra Filho et al., 1975; Karl et al., 1988) ou não antrópica como a intensificação da atividade solar e fenômenos naturais como o El Niño e La Niña (Marengo, 2010).

Alguns estudos com o objetivo de detectar possíveis tendências temporais aplicaram satisfatoriamente o método de Mann-Kendall em variáveis ambientais, entre eles: nível de água (Zhang et al., 2009), temperatura média (Karmeshu, 2012), precipitação (Moraes et al., 1998; Groppo et al., 2005; Karmeshu, 2012), cobertura vegetal (Neeti \& Eastman, 2011), qualidade de água (Groppo et al., 2008) e vazão (Douglas et al., 2000; Groppo et al., 2005, 2008).

A identificação dos locais que estão sofrendo alguma tendência meteorológica possibilita estimar os possíveis danos destas mudanças nas atividades socioeconômicas, auxiliando os tomadores de decisão a respeito do risco caso nenhuma ação seja tomada.

Diante do exposto, o objetivo do presente trabalho foi calcular e regionalizar tendências temporais significativas em séries históricas entre os anos de 1961 e 2011, para precipitação, temperatura média, umidade relativa e evapotranspiração potencial no Brasil utilizando o método Contextual Mann-Kendall (CMK), em todo território brasileiro.

\section{Metodologia}

\subsection{Descrição dos dados}

Pela falta de rede meteorológica de boa qualidade no Brasil, optou-se no presente trabalho utilizar mapas interpolados pelo Climatic Research Unit - CRU (University of East Anglia Climatic Research Unit, 2012) da University of East Anglia, localizada em Norwich no Reino Unido. Estes mapas foram escolhidos devido ao rigor na definição do banco de dados a ser utilizado (New et al., 2000) e na metodologia da interpolação dos mesmos (New et al., 1999), na qual entre outros fatores foi considerada a altitude de cada localidade. Os mapas interpolados possuem uma frequência mensal, com resolução espacial de $0.5^{\circ}$ de longitude por $0.5^{\circ}$ de latitude para toda área continental da Terra exceto a Antártida. A sua última versão (Harris et al., 2013) abrange o período entre os anos de 1901 e 2011.

Para o presente estudo, foram utilizadas séries mensais entre os anos de 1961 e 2011. Este período foi escolhido por coincidir com os dados medidos por estações meteorológicas do Instituto Nacional de Meteorologia (INMET), sendo possível conferir a consistência dos dados interpolados da precipitação e da temperatura pelo $C R U$.

Para isso, foram extraídos dados de 290 estações meteorológicas do INMET. Assim, para a temperatura média e a precipitação foram calculadas a correlação entre as séries do CRU e do INMET utilizando o método da regressão linear.

A umidade relativa, diferentemente das outras variáveis que foram obtidas diretamente do CRU, foi calculada a partir dos mapas interpolados de pressão de vapor $(e) \mathrm{e}$ temperatura média $\left(t_{m e d}\right)$. A pressão de vapor de saturação $\left(e_{s}\right)$, em milibares $(\mathrm{mb})$, foi calculada utilizando-se a equação de Tétens (Saucier, 1955), Eq. (1), para $T_{\text {med }}-50<T<$ $100{ }^{\circ} \mathrm{C}$ (condição satisfeita para todos os pontos em todos os meses para a região de estudo), cuja única variável é a temperatura média em graus Celsius. A umidade relativa $(U R)$ é a razão entre a pressão de vapor $(e)$ e a pressão de vapor saturada $\left(e_{s}\right)$, Eq. (2).

$$
\begin{aligned}
& e_{s}=6,1078 \times 10^{\left(\frac{17,27 t_{\text {med }}}{237,3+t_{\text {med }}}\right)} \\
& U R=\frac{e}{e_{s}} \times 100
\end{aligned}
$$

\subsection{Método de Mann-Kendall e CMK}

O Teste de Mann-Kendall (Mann, 1945; Kendall, 1975) é um método robusto, sequencial e não paramétrico utilizado para determinar se determinada série de dados possui uma tendência temporal de alteração estatisticamente significativa. Por tratar-se de um método não paramétrico, ele não requer distribuição normal dos dados (Yue et al., 2002). Outra vantagem deste método é o fato de ser pouco influenciado por mudanças abruptas ou séries não homogêneas (Zhang et al., 2009). No entanto, este método exige que os dados sejam independentes e aleatórios (Neeti \& Eastman, 2011).

A verificação da independência da série temporal foi realizada através do teste de autocorrelação serial e para a aleatoriedade dos dados foi realizado através do teste de sequência (Run Test).

O método baseia-se em rejeitar ou não a hipótese nula $\left(H_{0}\right)$, de que não exista tendência na série de dados, adotando-se um nível de significância $(\alpha)$. O nível de significância pode ser interpretado como a probabilidade de cometer-se o erro de rejeitar a $H_{0}$ quando esta for verdadeira. 
A variável estatística $S$, para uma série de $n$ dados do teste de Mann-Kendall é calculada a partir da somatória dos sinais (sgn) da diferença, par a par, de todos valores da série $\left(x_{i}\right)$ em relação aos valores que a eles são futuros $\left(x_{j}\right)$, expressa nas Eqs. (3) e (4).

$$
\begin{aligned}
& S=\sum_{i=1}^{n-1} \sum_{j=i+1}^{n} \operatorname{sgn}\left(x_{j}-x_{i}\right) \\
& \operatorname{sgn}\left(x_{j}-x_{i}\right)=\left\{\begin{array}{r}
+1 ; \text { se } x_{j}>x_{i} \\
0 ; \text { se } x_{j}=x_{i} \\
-1 ; \text { se } x_{j}<x_{i}
\end{array}\right.
\end{aligned}
$$

Quando $n \geq 10$, a variável $S$ pode ser comparada com uma distribuição normal, na qual a sua variância, $\operatorname{Var}(S)$, pode ser obtida através da Eq. (5), na qual $t_{i}$ representa a quantidade de repetições de uma extensão $i$ (Por exemplo, uma série histórica com três valores iguais entre si teria 1 repetição de extensão igual a 3 , ou $t_{i}=1$ e $i=3$ ).

$$
\operatorname{Var}(S)=\frac{n(n-1)(2 n+5)-\sum_{i=1}^{n} t_{i}(i)(i-1)(2 i+5)}{18}
$$

$\mathrm{O}$ indicie $Z_{M K}$ segue a distribuição normal, na qual a sua média é igual a zero, valores positivos indicam uma tendência crescente e negativos tendências decrescentes. De acordo com o sinal de $S$, o indicie $Z_{M K}$ da distribuição normal é calculado a partir da Eq. (6):

$$
\begin{aligned}
& Z_{M K}=\frac{S-1}{\sqrt{\operatorname{Var}(S)}} ; \text { para } S>0 \\
& Z_{M K}=0 ; \text { para } S=0 \\
& Z_{M K}=\frac{S+1}{\sqrt{\operatorname{Var}(S)}} ; \text { para } S<0
\end{aligned}
$$

Por se tratar de um teste bi-caudal, para rejeitar a $H_{0}$ é preciso que o valor absoluto de $Z_{M K}$ seja superior a $Z_{\alpha} / 2$. Por exemplo, para $\alpha=5 \%, Z_{0,05 / 2}=Z_{0,025}=1,96$ portanto será considerada que a série tenha uma tendência significativa ao nível de $5 \%$ caso $Z_{M K}>1,96$.

Após a aplicação do teste de Mann-Kendall, foi aplicado o teste Contextual Mann-Kendall (CMK) (Neeti e Eastman, 2011). Um dos princípios fundamentais da Geografia é que regiões vizinhas tendem a possuir características semelhantes. No entanto, o teste de Mann-Kendall é realizado individualmente em cada pixel, sendo possível que pixels vizinhos tenham comportamentos opostos, fato que raramente será verdadeiro.

O método CMK consiste na regionalização da série aplicando-se uma máscara da média com dimensão " $3 \times 3$ " após o cálculo da variável "S" do teste de Mann-Kendall.

\subsection{SIG e cálculos}

Os dados foram compilados e analisados utilizando-se o software de Sistema de Informação Geográfica (SIG) Idrisi Selva 17.02. Através do seu módulo Earth Trends Modeler (ETM) é possível analisar tendências em séries históricas espacialmente distribuídas, assim como comparar séries.

Para evitar a auto-correlação nas séries históricas, anteriormente à aplicação do teste $\mathrm{CMK}$, foi realizado uma processamento nos dados chamado Prewhitening presente no módulo ETM do Idrisi. O processamento Prewhitening consiste numa série cálculos iterativos através de ajustes na variância dos dados para compensar a possível existência de uma correlação temporal na série histórica (Wang \& Swail, 2001).

Os níveis de significância utilizados foram 1, 5 e $10 \%$. Para cada um dos níveis de significância foi considerado o sinal, positivo ou negativo, do " $Z$ " de MannKendall, Eq. (6), para concluir se no pixel a tendência é, respectivamente, positiva ou negativa.

\section{Resultados}

Os dados do CRU apresentaram boa correlação com os dados medidos pelo INMET, o coeficiente de determinação médio $\left(R^{2}\right)$ entre todas as estações foi de $0,81 \mathrm{com}$ um desvio padrão de 0,09 para precipitação (Figura 1) e 0,88 com um desvio padrão de 0,11 para a temperatura média (Figura 2), garantindo assim a consistência dos dados interpolados pelo CRU, tornando viável a sua utilização para uma análise de tendências no Brasil. Os piores

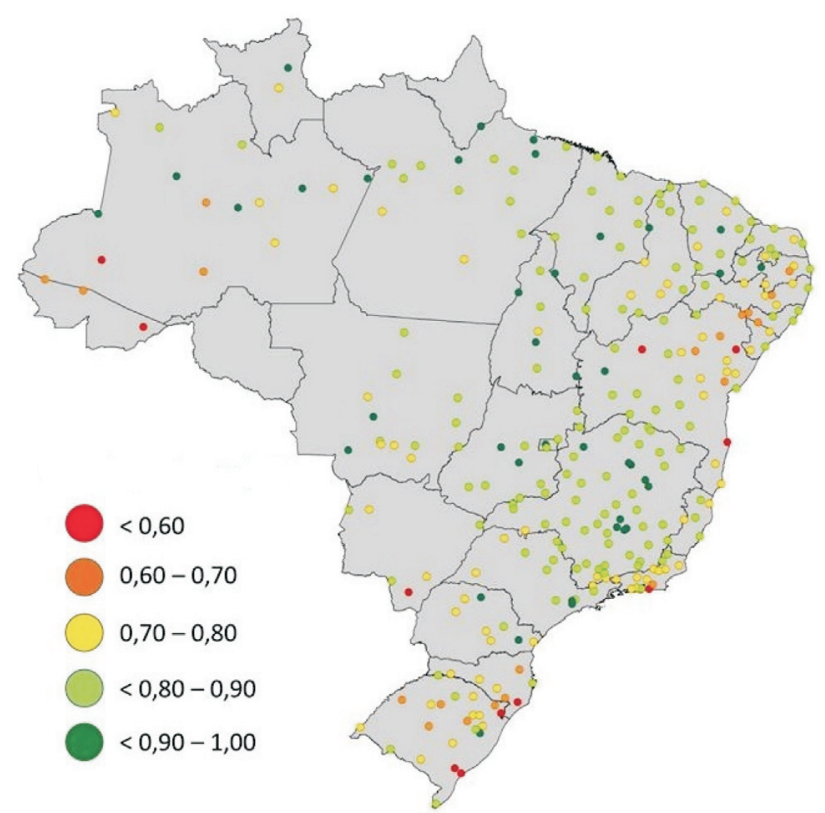

Figura 1 - Mapa do coeficiente de determinação $\left(\mathrm{R}^{2}\right)$ dos dados interpolados pelo CRU com os dados observados pelas estações do INMET para a Precipitação. 


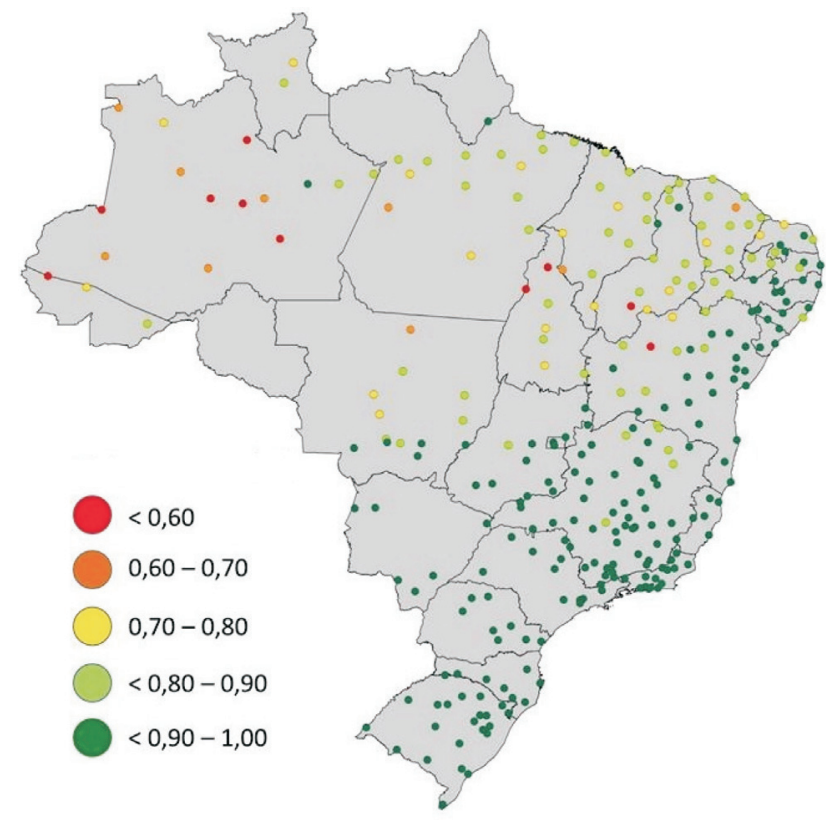

Figura 2 - Mapa do coeficiente de determinação $\left(\mathrm{R}^{2}\right)$ dos dados interpolados pelo CRU com os dados observados pelas estações do INMET para a Temperatura média.

coeficientes foram obtidos na região norte do Brasil, onde possui o menor número de estações meteorológicas disponíveis.

\subsection{Precipitação}

A precipitação (Figura 3) não apresentou tendências significativas em mais de $70 \%$ do território brasileiro em todos os meses, com tendências não significativas variando entre 72 e $95 \%$ nos meses de março e fevereiro, respectivamente (Tabela 1). O oeste da Amazônia apresentou tendência positiva no período mais chuvoso (janeiro a abril) e negativa no período mais seco (junho a setembro). Em contrapartida, nos meses mais secos foram constatadas tendências positivas na região da Nordeste, principalmente no semiárido, e nos estados de Mato Grosso e Goiás. O mês de janeiro apresentou tendências positivas na faixa litorânea entre Rio Grande do Sul e Rio de Janeiro.

\subsection{Temperatura média}

A maior parte do Brasil apresentou tendências positivas para temperatura média ao longo do ano inteiro (Figura 4), com áreas com tendências positivas de significância de $5 \%$ variando entre 36 e $78 \%$ do território brasileiro (Tabela 2). As regiões do norte da Caatinga, oeste da Amazônia e do Centro-Oeste, leste de Minas Gerais e os estados do Rio de Janeiro e Espírito Santo não apresentaram tendência positiva em alguns meses do ano.

\subsection{Evapotranspiração potencial}

Para a maior parte do território brasileiro, as tendências da evapotranspiração potencial (Figura 5) apresen- taram uma relação direta com a temperatura média, com alta porcentagem de tendências positivas de significância de 1\% (Tabela 3). Em regiões do Centro-Oeste, tendências positivas de evapotranspiração se refletiram em tendências positivas de precipitação. Concordantemente com as outras variáveis, as regiões oeste e leste da Amazônia brasileira apresentaram comportamentos distintos.

\subsection{Umidade relativa}

Em geral a umidade relativa (Figura 6) apresentou um comportamento inverso à temperatura média, sendo que mais de $60 \%$ do território brasileiro não apresentou tendências significativas (Tabela 4). No oeste da Amazônia e dos estados do Mato Grosso e Mato Grosso do Sul foi encontrada tendências positivas, concordando com o aumento de chuva nestas regiões. Em contrapartida o leste da Amazônia apresentou tendências negativas.

\section{Discussão}

O Brasil, de uma maneira geral, apresenta uma boa disponibilidade hídrica. Porém, por suas dimensões continentais e diversidade climática, o país apresenta situações bastante distintas quanto à disponibilidade hídrica, sendo afetado tanto pela escassez hídrica, quanto pela degradação dos recursos causada pela poluição de origem doméstica e industrial (Tucci, 2001; ANA, 2013).

A região Nordeste, onde predomina o clima semiárido, é a região brasileira que apresenta a menor disponibilidade hídrica (Rodrigues Silva, 2004). Estudos realizados nessa região mostram resultados distintos em relação à tendência da precipitação. No presente trabalho foram encontradas tendências negativas somente no mês de março e tendências positivas entre os meses de julho e dezembro na região da Nordeste, principalmente no semiárido, e nos Estados de Mato Grosso e Goiás. O que pode ser atribuído pelo aumento da evapotranspiração e da umidade observado em praticamente em todos os meses, como observado nas Figuras 3 e 4, respectivamente.

Os resultados obtidos na precipitação da região oeste da Amazônia, com tendência positiva no período mais chuvoso (janeiro a abril) e negativa no período mais seco (junho a setembro), pode indicar acentuação dos extremos.

Resultados similares com o aumento na precipitação no Estado do Ceará, situado na região Nordeste do Brasil, também foram encontrados por Wagner (1996) e Hastenrath (2000) e por Santos et al. (2010). O deslocamento da Zona de Convergência Intertropical (ZCIT), pode ser a explicação das tendências positivas de chuva na região Nordeste (Hastenrath, 2001). O período de estudo pode ter sido o responsável pelos resultados distintos encontrados por Moncunill (2006), que observou redução da chuva total anual da ordem de $6 \%$ por década no período entre $1961 \mathrm{e}$ 2003 para quase todo Estado do Ceará, com exceção em regiões onde a precipitação é dependente da topografia, e 


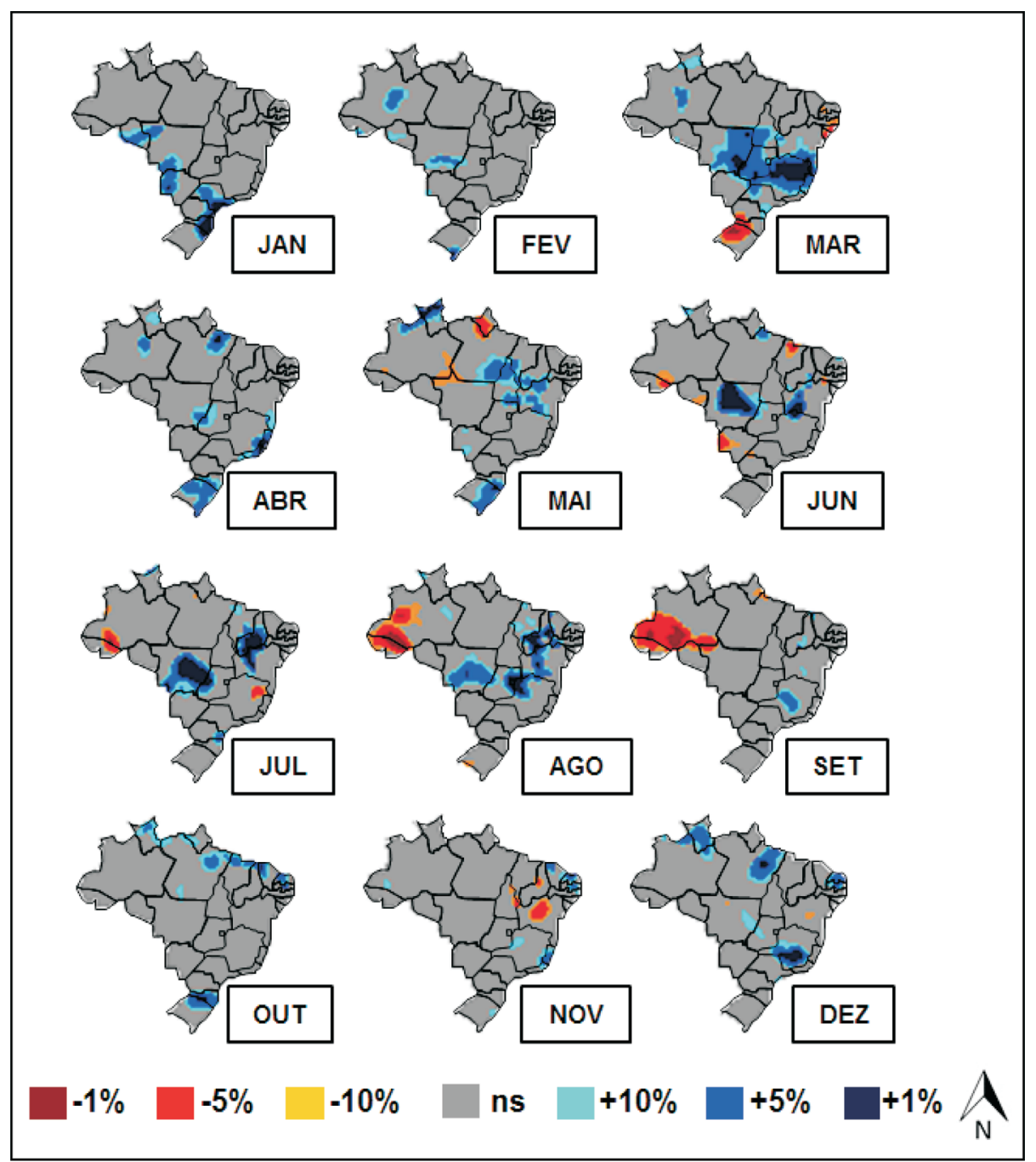

Figura 3 - Mapas mensais de tendências de Precipitação classificados em áreas sem tendência significativa (cor cinza) e com tendências significativas positivas (tons de azul) e negativas (tons de vermelho) para os níveis de significância (1, 5 e 10\%).

Tabela 1 - Porcentagem da área do Brasil, para Precipitação, com tendências, positivas e negativas, para níveis de significância de $10 \%, 5 \%$ e $1 \%$.

\begin{tabular}{lccccccc}
\hline \multirow{2}{*}{ Mês } & \multicolumn{7}{c}{ Níveis de significância } \\
\cline { 2 - 8 } & $+1 \%$ & $+5 \%$ & $+10 \%$ & $\begin{array}{c}\text { Não } \\
\text { significativa }\end{array}$ & $-10 \%$ & $-5 \%$ & $-1 \%$ \\
\hline Jan & $1,8 \%$ & $7,1 \%$ & $10,3 \%$ & $89,7 \%$ & $0,0 \%$ & $0,0 \%$ & $0,0 \%$ \\
Fev & $0,1 \%$ & $1,9 \%$ & $4,8 \%$ & $95,2 \%$ & $0,0 \%$ & $0,0 \%$ & $0,0 \%$ \\
Mar & $4,7 \%$ & $17,3 \%$ & $24,4 \%$ & $72,1 \%$ & $3,4 \%$ & $2,3 \%$ & $0,5 \%$ \\
Abr & $0,7 \%$ & $6,0 \%$ & $11,3 \%$ & $88,7 \%$ & $0,0 \%$ & $0,0 \%$ & $0,0 \%$ \\
Mai & $0,9 \%$ & $8,6 \%$ & $15,2 \%$ & $81,3 \%$ & $3,5 \%$ & $0,9 \%$ & $0,0 \%$ \\
Jun & $3,8 \%$ & $6,5 \%$ & $9,2 \%$ & $87,5 \%$ & $3,3 \%$ & $1,1 \%$ & $0,1 \%$ \\
Jul & $5,2 \%$ & $9,3 \%$ & $12,9 \%$ & $84,8 \%$ & $2,4 \%$ & $1,4 \%$ & $0,2 \%$ \\
Ago & $3,0 \%$ & $9,3 \%$ & $14,3 \%$ & $78,9 \%$ & $6,8 \%$ & $4,0 \%$ & $1,5 \%$ \\
Set & $0,0 \%$ & $1,0 \%$ & $2,1 \%$ & $87,7 \%$ & $10,1 \%$ & $7,7 \%$ & $1,7 \%$ \\
Out & $0,4 \%$ & $4,0 \%$ & $8,9 \%$ & $91,1 \%$ & $0,0 \%$ & $0,0 \%$ & $0,0 \%$ \\
Nov & $0,1 \%$ & $0,9 \%$ & $2,7 \%$ & $94,9 \%$ & $2,4 \%$ & $1,0 \%$ & $0,0 \%$ \\
Dez & $1,4 \%$ & $7,2 \%$ & $11,8 \%$ & $88,0 \%$ & $0,2 \%$ & $0,0 \%$ & $0,0 \%$ \\
\hline
\end{tabular}

por Salati et al. (2007) que observaram uma diminuição de $11,6 \%$ na precipitação entre os anos de 1961 a 2004.

O Nordeste apresentou tendências positivas significativas na temperatura média somente na faixa litorânea, já na região semi-árida não foi observada nenhuma tendência. Santos et al. (2010) e Salati et al. (2007) também apresentaram tendências de positivas na temperatura do ar média anual e na evapotranspiração potencial em quase toda a região Nordeste.

Para a Região Centro-Oeste, Salati et al. (2007), apresentaram resultados similares ao presente estudo, com tendências de aumento na temperatura média na região. Marengo et al. (2010) realizaram projeções climáticas regionais, no qual todos os modelos apontaram para uma tendência de aquecimento para o final do século XXI para a região Centro-Oeste, especialmente durante a primavera e verão. Nestas estações, o incremento nas temperaturas médias variaram entre 4 e $5{ }^{\circ} \mathrm{C}$, dependendo do modelo. Quanto às projeções para as precipitações, os modelos 


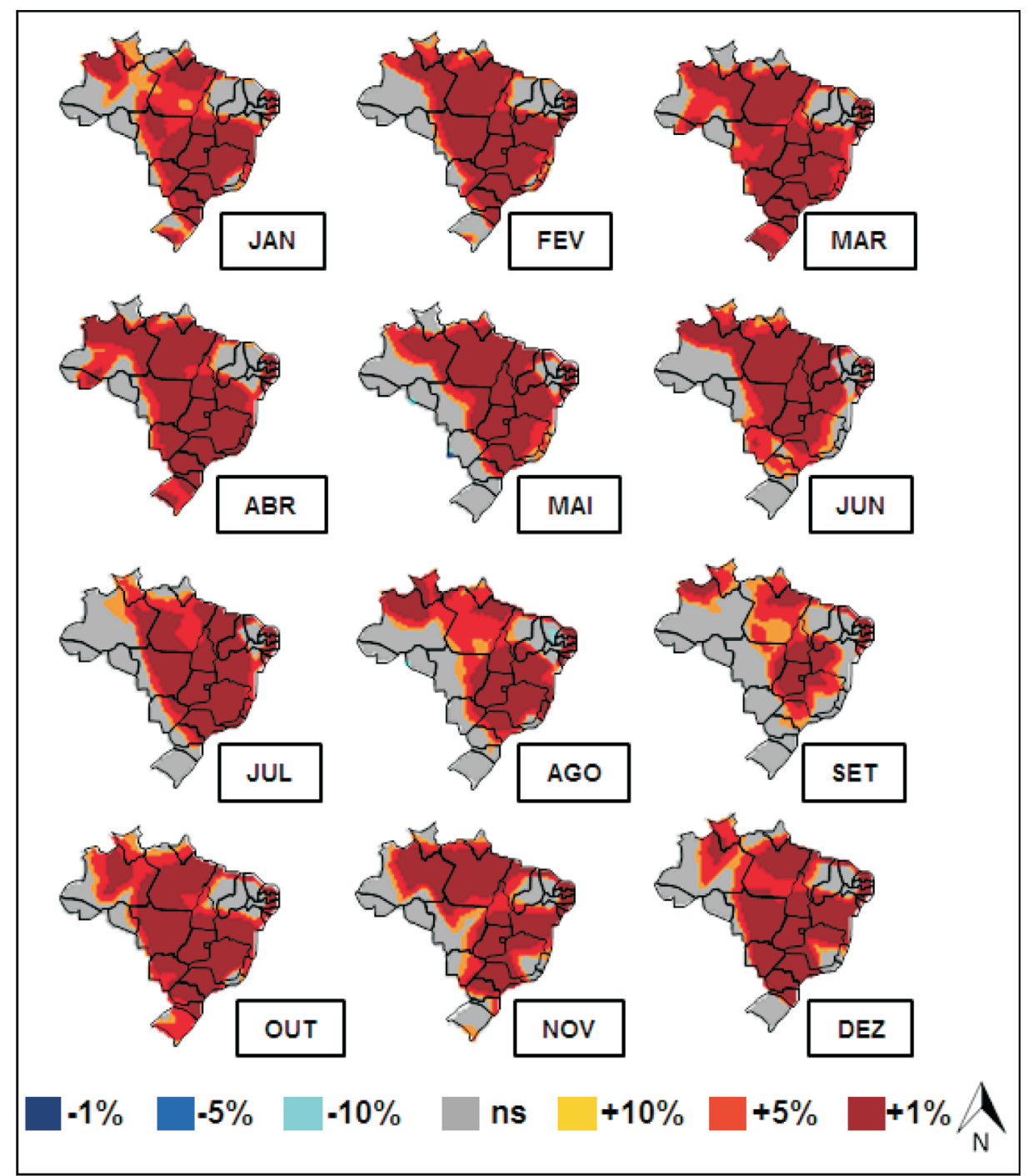

Figura 4 - Mapas mensais de tendências da Temperatura média, classificados em áreas sem tendência significativa (cor cinza) e com tendências significativas positivas (tons de vermelho) e negativas (tons de azul) para os níveis de significância (1, 5 e 10\%).

Tabela 2 - Porcentagem da área do Brasil, para Temperatura média, com tendências, positivas e negativas, para níveis de significância de $10 \%, 5 \%$ e $1 \%$.

\begin{tabular}{lrrrrrrr}
\hline \multirow{2}{*}{ Mês } & \multicolumn{7}{c}{ Níveis de significância } \\
\cline { 2 - 8 } & $+1 \%$ & $+5 \%$ & $+10 \%$ & $\begin{array}{c}\text { Não } \\
\text { significativa }\end{array}$ & $-10 \%$ & $-5 \%$ & $-1 \%$ \\
\hline Jan & $40,6 \%$ & $61,8 \%$ & $73,0 \%$ & $27,0 \%$ & $0,0 \%$ & $0,0 \%$ & $0,0 \%$ \\
Fev & $55,5 \%$ & $67,0 \%$ & $72,8 \%$ & $27,2 \%$ & $0,0 \%$ & $0,0 \%$ & $0,0 \%$ \\
Mar & $59,0 \%$ & $77,7 \%$ & $82,5 \%$ & $17,5 \%$ & $0,0 \%$ & $0,0 \%$ & $0,0 \%$ \\
Abr & $62,5 \%$ & $76,8 \%$ & $81,3 \%$ & $18,7 \%$ & $0,0 \%$ & $0,0 \%$ & $0,0 \%$ \\
Mai & $50,4 \%$ & $61,4 \%$ & $66,4 \%$ & $33,2 \%$ & $0,5 \%$ & $0,1 \%$ & $0,0 \%$ \\
Jun & $47,2 \%$ & $63,0 \%$ & $70,4 \%$ & $29,6 \%$ & $0,0 \%$ & $0,0 \%$ & $0,0 \%$ \\
Jul & $48,0 \%$ & $61,6 \%$ & $67,4 \%$ & $32,6 \%$ & $0,0 \%$ & $0,0 \%$ & $0,0 \%$ \\
Ago & $35,4 \%$ & $57,6 \%$ & $65,2 \%$ & $34,5 \%$ & $0,4 \%$ & $0,0 \%$ & $0,0 \%$ \\
Set & $18,4 \%$ & $35,9 \%$ & $49,6 \%$ & $50,4 \%$ & $0,0 \%$ & $0,0 \%$ & $0,0 \%$ \\
Out & $53,6 \%$ & $73,8 \%$ & $80,6 \%$ & $19,4 \%$ & $0,0 \%$ & $0,0 \%$ & $0,0 \%$ \\
Nov & $46,0 \%$ & $62,4 \%$ & $70,9 \%$ & $29,1 \%$ & $0,0 \%$ & $0,0 \%$ & $0,0 \%$ \\
Dez & $47,8 \%$ & $65,7 \%$ & $71,8 \%$ & $28,2 \%$ & $0,0 \%$ & $0,0 \%$ & $0,0 \%$ \\
\hline
\end{tabular}

apresentam grande divergência, apresentando tanto tendências negativas, quanto positivas (Marengo et al., 2010).

Tendências positivas também foram encontradas na temperatura média e na precipitação para região Norte (Salati et al., 2007) e tendência de crescimento da precipitação e de vazão do rio Negro desde o início do século XX na Amazônia, nas cidades de Belém e de Manaus (Cutrim et al., 2000). O aumento sistemático da convecção sobre o norte da Amazônia desde 1975 seria a possível explicação para o aumento de chuva na região (Chu et al., 1995).

A análise das diferenças entre as médias do período de 1991 a 2004 e do período de 1961 a 1990 para as temperaturas máximas, médias e mínimas, indicaram que para a Região Sudeste a temperatura média apresentou um aumento de $0,6^{\circ} \mathrm{C}$ nos períodos estudados e para as temperaturas máxima e mínima os valores indicaram um aumento de $0,4{ }^{\circ} \mathrm{C}$, para a precipitação houve um aumento de $57 \mathrm{~mm}$, o que representa um aumento de $4,8 \%$. Com rela- 


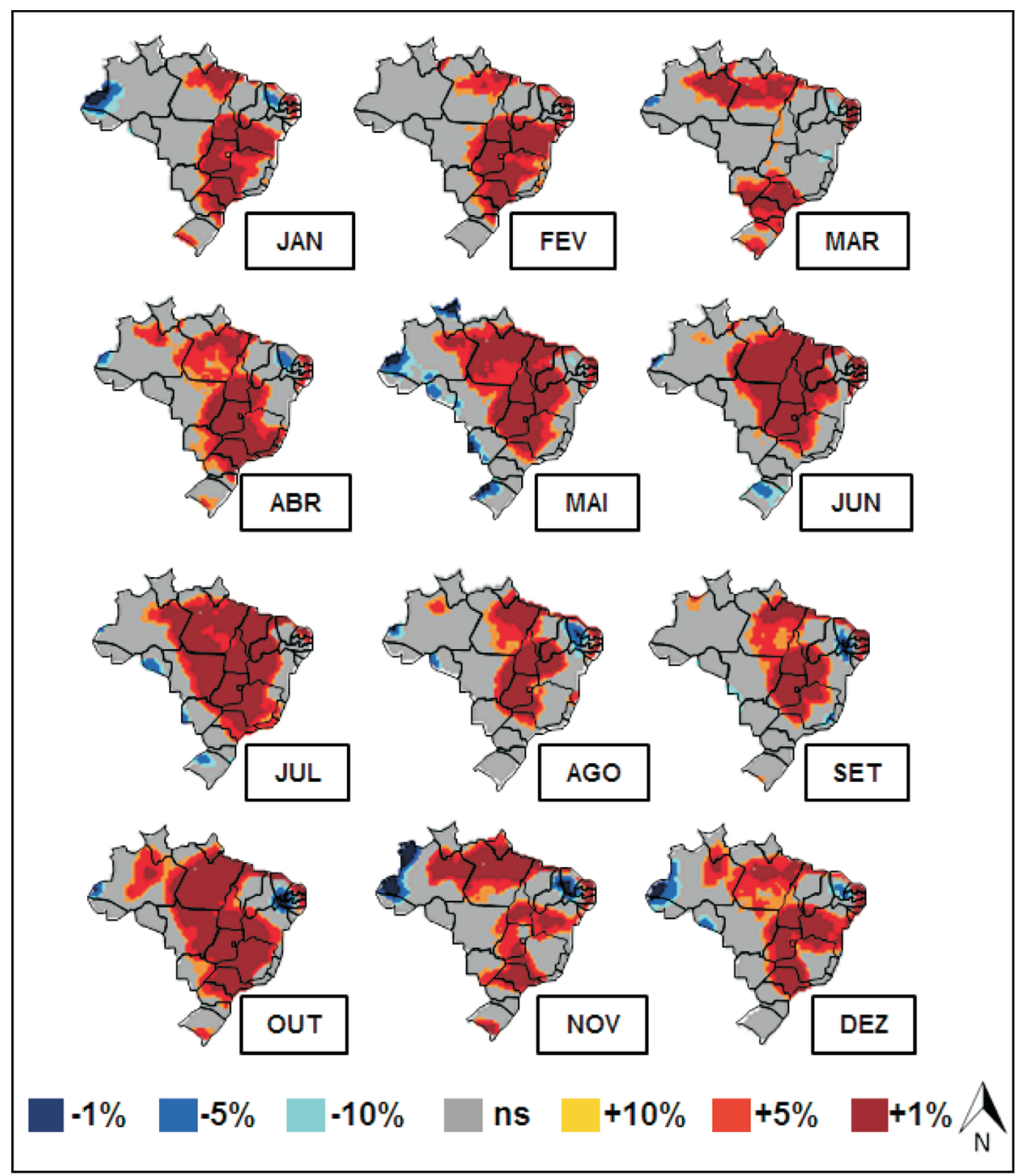

Figura 5 - Mapas mensais de tendências da Evapotranspiração Potencial, classificados em áreas sem tendência significativa (cor cinza) e com tendências significativas positivas (tons de vermelho) e negativas (tons de azul) para os níveis de significância (1, 5 e 10\%).

Tabela 3 - Porcentagem da área do Brasil, para Evapotranspiração Potencial, com tendências, positivas e negativas, para níveis de significância de $10 \%, 5 \%$ e $1 \%$

\begin{tabular}{lrrrrrrr}
\hline \multirow{2}{*}{ Mês } & \multicolumn{7}{c}{ Nícis de significância } \\
\cline { 2 - 8 } & $+1 \%$ & $+5 \%$ & $+10 \%$ & $\begin{array}{c}\text { Não } \\
\text { significativa }\end{array}$ & $-10 \%$ & $-5 \%$ & $-1 \%$ \\
\hline Jan & $19,1 \%$ & $29,3 \%$ & $34,1 \%$ & $60,5 \%$ & $5,4 \%$ & $3,7 \%$ & $1,5 \%$ \\
Fev & $20,6 \%$ & $30,8 \%$ & $36,4 \%$ & $63,6 \%$ & $0,0 \%$ & $0,0 \%$ & $0,0 \%$ \\
Mar & $14,8 \%$ & $26,9 \%$ & $32,6 \%$ & $66,2 \%$ & $1,2 \%$ & $0,4 \%$ & $0,0 \%$ \\
Abr & $23,0 \%$ & $39,2 \%$ & $49,5 \%$ & $48,6 \%$ & $1,9 \%$ & $1,2 \%$ & $0,0 \%$ \\
Mai & $32,2 \%$ & $45,5 \%$ & $49,6 \%$ & $41,1 \%$ & $9,4 \%$ & $6,0 \%$ & $2,4 \%$ \\
Jun & $34,1 \%$ & $41,4 \%$ & $46,2 \%$ & $51,5 \%$ & $2,3 \%$ & $1,2 \%$ & $0,2 \%$ \\
Jul & $42,0 \%$ & $52,1 \%$ & $56,4 \%$ & $41,1 \%$ & $2,6 \%$ & $1,4 \%$ & $0,0 \%$ \\
Ago & $19,3 \%$ & $27,7 \%$ & $33,9 \%$ & $62,8 \%$ & $3,3 \%$ & $2,2 \%$ & $0,5 \%$ \\
Set & $16,6 \%$ & $27,1 \%$ & $33,6 \%$ & $63,6 \%$ & $2,8 \%$ & $1,4 \%$ & $0,6 \%$ \\
Out & $39,1 \%$ & $52,8 \%$ & $59,9 \%$ & $37,3 \%$ & $2,7 \%$ & $2,1 \%$ & $1,1 \%$ \\
Nov & $22,8 \%$ & $37,2 \%$ & $43,8 \%$ & $49,0 \%$ & $7,2 \%$ & $6,4 \%$ & $4,0 \%$ \\
Dez & $20,1 \%$ & $36,0 \%$ & $45,2 \%$ & $49,0 \%$ & $5,8 \%$ & $3,8 \%$ & $1,1 \%$ \\
\hline
\end{tabular}

ção às precipitações, existe uma grande variabilidade nas mesmas regiões e observa-se que a perda de água por evapotranspiração é bastante elevada em grande parte do território nacional, sendo em várias regiões acima de $80 \%$, entre os menores valores encontrados está a Região Sudeste. Essa constatação indica que esta região é das mais críticas com referência à oferta de recursos hídricos, em função da variabilidade das suas precipitações (Salati et al., 2007). Groppo et al. (2005) também apresentaram tendências positivas na precipitação em algumas bacias hidrográficas distribuídas no Estado de São Paulo entre os anos de 1947-1997.

Marengo e Camargo (2008), estudando as temperaturas máximas e mínimas no sul do Brasil, durante o período 1960-2002, encontraram um aquecimento sistemático da região Sul, detectando tendências positivas na temperatura máxima e mínima a níveis anual e sazonal. Salati et al. (2007) apresentaram um aumento na temperatura média e na precipitação. Ainda em relação ao regime 


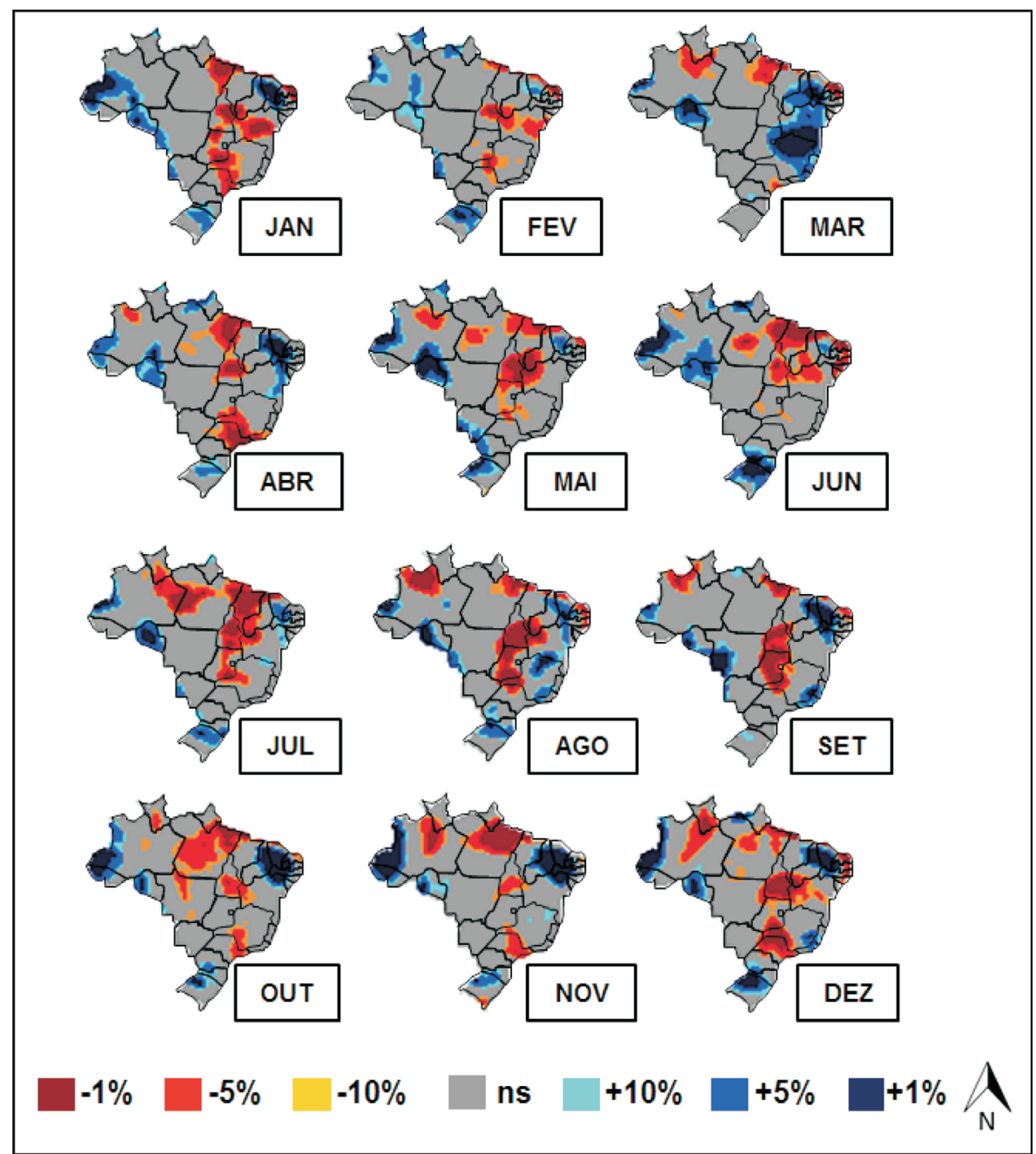

Figura 6 - Mapas mensais de tendências da Umidade Relativa, classificados em áreas sem tendência significativa (cor cinza) e com tendências significativas positivas (tons de vermelho) e negativas (tons de azul) para os níveis de significância (1, 5 e $10 \%$ ).

Tabela 4 - Porcentagem da área do Brasil, para Umidade Relativa, com tendências, positivas e negativas, para níveis de significância de 10\%,5\% e $1 \%$.

\begin{tabular}{lccccccc}
\hline \multirow{2}{*}{ Mês } & \multicolumn{7}{c}{ Nívis de significância } \\
\cline { 2 - 8 } & $+1 \%$ & $+5 \%$ & $+10 \%$ & $\begin{array}{c}\text { Não } \\
\text { significativa }\end{array}$ & $-10 \%$ & $-5 \%$ & $-1 \%$ \\
\hline Jan & $4,8 \%$ & $11,6 \%$ & $15,8 \%$ & $68,0 \%$ & $16,2 \%$ & $12,7 \%$ & $6,0 \%$ \\
Fev & $10,3 \%$ & $15,5 \%$ & $20,9 \%$ & $70,5 \%$ & $8,6 \%$ & $5,2 \%$ & $1,1 \%$ \\
Mar & $8,3 \%$ & $18,4 \%$ & $22,6 \%$ & $68,5 \%$ & $8,9 \%$ & $5,8 \%$ & $1,2 \%$ \\
Abr & $2,7 \%$ & $10,4 \%$ & $15,5 \%$ & $69,3 \%$ & $15,2 \%$ & $10,7 \%$ & $4,8 \%$ \\
Mai & $4,7 \%$ & $10,7 \%$ & $14,6 \%$ & $67,5 \%$ & $17,8 \%$ & $11,7 \%$ & $3,3 \%$ \\
Jun & $5,3 \%$ & $13,4 \%$ & $17,2 \%$ & $65,8 \%$ & $17,0 \%$ & $11,7 \%$ & $4,8 \%$ \\
Jul & $2,0 \%$ & $6,9 \%$ & $11,0 \%$ & $67,0 \%$ & $22,0 \%$ & $17,5 \%$ & $8,4 \%$ \\
Ago & $3,3 \%$ & $10,6 \%$ & $15,0 \%$ & $67,4 \%$ & $17,6 \%$ & $14,6 \%$ & $9,3 \%$ \\
Set & $5,1 \%$ & $9,7 \%$ & $13,2 \%$ & $73,1 \%$ & $13,7 \%$ & $10,7 \%$ & $5,6 \%$ \\
Out & $7,0 \%$ & $11,7 \%$ & $14,6 \%$ & $65,6 \%$ & $19,8 \%$ & $12,1 \%$ & $2,0 \%$ \\
Nov & $9,2 \%$ & $13,0 \%$ & $16,5 \%$ & $67,3 \%$ & $16,2 \%$ & $12,0 \%$ & $5,5 \%$ \\
Dez & $7,8 \%$ & $12,9 \%$ & $16,2 \%$ & $59,7 \%$ & $24,1 \%$ & $16,7 \%$ & $7,2 \%$ \\
\hline
\end{tabular}

de chuvas, Groissman et al. (2005) identificaram tendências positivas de aumento sistemático de chuva e de extremos de chuva na região Sul.

Mudança no ciclo hidrológico, ocasionadas pelo possível aumento da temperatura no futuro (Salati et al., 2007; Marengo et al., 2010), gerando um provável aumento nas taxas de evaporação de lagos e reservatórios, o que causaria uma redução na oferta hídrica. Por outro lado, o aumento nas taxas de evapotranspiração, ainda se mostra uma questão em aberto, principalmente por causa do efeito do aumento do $\mathrm{CO}_{2}$ no comportamento dos estômatos e na densidade e distribuição espacial da vegetação (Pinto, 2009).

\section{Conclusões}

O fato de todas variáveis estudadas apresentarem tendências significativas em todos os meses indica que mudanças importantes estão ocorrendo, principalmente em relação à temperatura média. Estudos mais aprofundados 
são necessários para determinar as causas destas mudanças, porém a continuidade dessas tendências poderá resultar em impactos na agricultura, no ciclo hidrológico, e consequentemente para a fauna e flora e para a população.

\section{Referências}

ANA, Agência Nacional de Águas (Brasil). Conjuntura dos recursos hídricos no Brasil: 2013 - Brasília: ANA, 432p, 2013.

CHU, P. S.; YU, P.; HASTENRATH, S. Bulletin of the American Meteorological Society, 75, 1995.

CUTRIM E.M.C; MOLION L.C.B.; NECHET, D. Chuvas na Amazônia durante o século XX. Anais: XI Congresso Brasileiro de Meteorologia. Rio de Janeiro. 1327-1333. 2000.

DE LUCENA, A.F.P; SCHAEFFER, R; SZKLO, A. A Vulnerabilidade do Sistema de Energia Elétrica à Mudança Climática no Brasil. FBDS (org) Mudanças Climáticas Eventos Extremos no Brasil. p: 20-33. FDBS \& LLOYD'S. 2010.

DOUGLAS, E.M; VOGEL, R.M; KROLL, C.N. Trends in floods and low flows in the United States: impact of spatial correlation. Journal of Hydrology 240:90-105. 2000.

GROISSMAN, P; KNIGHT, P; EASTERLING, D; KARL, T; HEGERL, G;RAZUVAEK, V. Trends in intense precipitation in the climate record. Journal of Climate18:1326-1350. 2005.

GROPPO, J.D.; MORAES, J. M.; BEDUSCHI, C. E. ; MARTINELLI, L. A. Trend analysis of water quality in some rivers with different degrees of development within the Sao Paulo State, Brazil. Rivers Research and Applications, v. 1, p. $1-2,2008$.

GROPPO, J.D., BEDUSCHI, C. E., MENUZZO, R., MORAES, J. M., MARTINELLI, L. A. Análise de séries temporais de vazão e precipitação em algumas bacias do estado de São Paulo com diferentes graus de intervenções antrópicas. Revista Brasileira de Geociências. , v.24, p.181 - 192, 2005.

HARRIS, I; JONES, P.D; OSBORN, T.J; LISTE, D.H. Updated high-resolution grids of monthly climatic observations - the CRU TS3.10 Dataset. International Journal of Climatology. 2013.

HASTENRATH, S., Interannual and Longer-Term Variability of Upper Air Circulation in the Northeast Brazil-Tropical Atlantic Sector, Journal of Geophysical Research, 105, No. D6, 7327-7335, 2000.

Intergovernmental Panel on Climate Change - IPCC. Climate Change 2007: The Physical Science Basis. Contribution of Working Group I to the Fourth Assessment Report of the Intergovernmental Panel on Climate Change Solomon, S., D. Qin, M. Manning, Z. Chen, M. Marquis, K. B. Averyt, M. Tignor and H. L. Miller Eds., Cambridge University Press, Cambridge, United Kingdom and New York, NY, USA, 996 pp. 2007.

KARL, T.R.; DIAZ, H.F.; KUKLA, G. Urbanization: its detection and effect in the United States climate record. Journal of Climate, v.1 n.11, p.1099-1123. 1988.

KARMESHU, N. Trend Detection in Annual Temperature \& Precipitation using the Mann Kendall Test - A Case Study to Assess Climate Change on Select States in the Northeastern United States. Master of Environmental Studies Capstone
Projects. University of Pennslvania. Department of Earth and Environmental Science. August, 2012.

KENDALL, M.G.. Rank Correlation Methods. Charles Griffin, London. 1975

MANN, H.B. Nonparametric tests against trend. Econometrica 13, 245-259. 1945.

Marengo, J. A. and CAMARGO, C. C. Surface air temperature trends in Southern Brazil for 1960-2002. Int. J. Climatol., 28:893-904, 2008.

MARENGO, J. A. Mudanças Climáticas, Condições Meteorológicas Extremas e Eventos Climáticos no Brasil. FBDS (org) Mudanças Climáticas Eventos Extremos no Brasil. p: 05-19. FDBS \& LLOYD'S. 2010.

MARENGO,J. A., TOMASELLA,J., UVO,C. R. B.- On the Suitability of Non-Parametric Tests for Detection of Trends in Brasilian Rivers, Congresso Brasileiro Metereologia, 1492-1495, 1996.

MONCUNILL, D.F. The rainfall trend over Ceará and its implications. Proceeding of 8 ICSHMO, Foz Iguaçu, Brazil INPE. 315-323pp. 2006.

MORAES, J.M; PELLEGRINO, G.Q; BALLESTER, M.V; MARTINELLI, L.A; VICTORIA, R.L; KRUSCHE, A.V. Trends in Hydrological Parameters of a Southern Brazilian Watershed and its Relation to Human Induced Changes. Water Resources Management. 12: 295-311, 1998.

NEETI, N; EASTMAN, J.R. A Contextual Mann-Kendall Approach for the Assessment of Trend Significance in Image Time Series. Transactions in GIS, 15(5): 599-611. 2011.

NEW, M; HULME, M; JONES, P. Representing TwentiethCentury Space-Time Variability. Part I: Development of a 1961-90 Mean Monthly Terrestrial Climatology. American Meteorological Society, 1999.

NEW, M; HULME, M; JONES, P. Representing Twentieth-Century Space-Time Climate Variability. Part II: Development of 1901-96 Monthly Grids of Terrestrial Surface Climate. American Meteorological Society, 2000.

NOBRE, C; YOUNG, A. F. ; SALDIVA, P. H. N. ;MARENGO, J. A.; NOBRE, A. D. ; OGURA, A. T. ; THOMAZ, O. ; OBREGON, G.; MOREIRA DA SILVA, G. C. ; VALVERDE, M. ; SILVEIRA, A. C. ; RODRIGUES, G. O. . Vulnerability of Brazilian Megacities to Climate Change: the São Paulo Metropolitan Region (RMSP). Climate Change in Brazil: economic, social and regulatory aspects. Brasilia: IPEA., v. , p. 197-219. 2011.

PBMC. Primeiro Relatório de Avaliação Nacional do Painel Brasileiro de Mudanças Climáticas. Sumário Executivo do GT2. PBMC, Rio de Janeiro, Brasil. 28 p. 2013.

PINTO, H.S; ASSAD, E.D; ZULLO, J.R; BRUNINI, O; EVANGELISTA, B.A. Impacto do Aumento da Temperatura no Zoneamento Climático do Café nos Estados de São Paulo e Goiás. Avaliação dos cenários do IPCC. XII Congresso Brasileiro de Agrometeorologia; pp: 605-606. Fortaleza. 2001.

PINTO, H. S.. Adaptação do Setor Agrícola Brasileiro. In: Fundação Brasileira para o Desenvolvimento Sustentável FBDS. (Org.). Mudanças Climáticas e Eventos Extremos no Brasil. Rio de Janeiro: DaGema Comunicação, v. 1, p. 34-51. 2009.

RODRIGUES DA SILVA, V.P. On climate variability in Northeast of Brazil. Journal of Arid Environments 58, 575-596. 2004. 
SALATI, E; SALATI, E; CAMPANHOL, T.; VILLA NOVA, N. Caracterização do clima atual e definição das alterações climáticas para o território brasileiro ao longo do Século XXI. Relatório 4. Tendências de Variações Climáticas para o Brasil no Século XX e Balanços Hídricos para Cenários Climáticos para o Século XXI. 182p. 2007.

SANTOS, D. N.; SILVA V. P. R.; SOUSA, F. A. S.; SILVA, R. A. Estudo de alguns cenários climáticos para o Nordeste do Brasil. Revista Brasileira de Engenharia Agrícola e Ambiental, v.14, n.5, p.492-500. 2010.

SAUCIER, W. J. Principles of meteorological analysis. Chicago: Universiy of Chicago Press. 1955.

SERRA FILHO, R.; CAVALLI, A.C.; GUILLAUMON, J.R.; CHIARINI, J.V.; NOGUEIRA, F.P.; IVANCKO, C.M.A.M.; BARBIERI, J.L.; DONIZELI, P.L.; COELHO, A.G.S.; BITTENCOURT, I. Levantamento da cobertura vegetal natural e do reflorestamento no Estado de São Paulo. Boletim Técnico do Instituto Florestal, v.11, p.1-56. 1975.

TUCCI, C. E. M. Gestão da água no Brasil - Brasília : UNESCO, 156p. 2001
UNIVERSITY OF EAST ANGLIA CLIMATIC RESEARCH UNIT (CRU). [Phil Jones, Ian Harris]. CRU Time Series (TS) high resolution gridded data version 3.20, [Internet]. NCAS British Atmospheric Data Centre, 2012, Disponível em abril de $\quad 2013$ em http://badc.nerc.ac.uk/view/badc.nerc.ac.uk_ATOM_AC TIVITY 3ec0d1c6-4616-11e2-89a3-00163e251233.

WAGNER, R., Decadal-scale trend in mechanims controlling meridional sea surface temperature gradients in the tropical Atlantic. J. Geophys. Res. V.101, p.16683-694. 1996.

WANG, X.L; SWAIL, V.R. Changes of extreme wave heights in Northern Hemisphere oceans and related atmospheric circulation regimes. Journal of Climate 14: 2204-21. 2001.

YUE, S; PILON, P; CAVADIAS, G. Power of the Mann-Kendall and Spearman's rho tests for detecting monotonic trends in hydrological series. Journal of Hydrology 259:254-271. 2002.

ZHANG, W; YAN, Y; ZHENG, J; LI, L; DONG, X; CAI, H. Temporal and spatial variability of annual extreme water level in the Pearl River Delta region, China. Global and Planetary Change 69:35-47. 2009.

All the contents of this journal, except where otherwise noted, is licensed under a Creative Commons Attribution License CC-BY. 